\title{
Mamíferos y aves silvestres usados por los pobladores de la cuenca del río Abujao (Ucayali, Perú)
}

\author{
Wild Mammals and birds used by inhabitants of the Abujao river basin (Ucayali, Peru)
}

\section{Jorge Washinton Vela Alvarado ${ }^{1}$, Manuel Rivas ${ }^{1}$, Victor Fernández ${ }^{1}$ y Mirella Clavo Peralta ${ }^{2}$}

1. Universidad Nacional de Ucayali - UNU, Facultad de Ciencias Agropecuarias, Centro de Investigación de Fronteras Amazónicas, CIFA. Pucallpa, Perú. 2. Universidad Nacional Mayor de San Marcos, Facultad de Medicina Veterinaria, Instituto Veterinario de Investigaciones Tropicales y de Altura, Estación Experimental IVITA, Pucallpa.

Email Jorge Vela Alvarado: jvelaunu@gmail.com

Email Manuel Rivas: derivas_321@hotmail.com

Email Victor Fernández: vicferde@hotmail.com

Email Mirella Clavo Peralta: zclavop@unmsm.edu.pe

\section{Resumen}

La Amazonia peruana esta clasificada como un ecosistema mega-diverso. La riqueza de especies de fauna y flora acrecienta la diversidad de la población humana. La cuenca del río Abujao está poblada por mestizos y grupos indígenas Ashéninka y Shipibo-Conibo, los cuales todavía guardan parte de sus tradiciones, conocimientos ancestrales y ecológicos. Esta investigación se realizó con la finalidad de conocer el uso de especies de mamíferos y aves silvestres por la población indígena y mestiza en la cuenca del río Abujao. Se determinaron categorías de uso; entre las categorías determinadas predomina el mayor conocimiento de mamíferos y aves silvestres para el uso alimenticio, medicinal y comercial; relativamente pocas especies y parte de estas son para uso ritual, mágico y ornamental debido a la pérdida de conocimientos y tradiciones ancestrales. Rescatando estos conocimientos el presente trabajo tiene una gran importancia en la conservación de aves y mamíferos y el conocimiento ecológico de la Amazonía Peruana.

Palabras claves: Amazonía; Etnozoología; Ashéninka; Shipibo-Conibo; categorías de uso; fauna silvestrel; Abujao; bosque tropical húmedo

\section{Abstract}

The Peruvian Amazon is classified as one of the mega-diverse ecosystem of the world. Local populations have benefited from the uses of its richness of fauna and flora, promoting the emergence of a wide variety of uses. The Abujao river basin, located in the Peruvian Amazon, is home for mestizos and indigenous groups of Ashéninka and Shipibo-Conibo, whose traditions, and ancestral and ecological knowledge are still alive and closely related to their natural environments. This research was carried out to determine how and to what extent present groups of indigenous and mestizo in the Abujao river basin have been using the wild species of mammals and birds in their locations. Categories of its uses were determined. Among of all defined categories, the most predominant one was the use of wild animals for human consumption, traditional medicine and commercial trades. In contrast, relatively few species, in whole or part, were still used for rituals, and ornamental due to the loss of some ancestral knowledge and traditions on these uses. Revaluing this set of knowledge and uses has a great importance in the conservation of birds and mammals as well as the ecological knowledge of local populations in the Peruvian Amazon.

Keywords: Amazon; Ethnozoology; Ashéninka; Shipibo-Conibo; mestizo; wild birds; wild mammals; Use categories; Abujao; Wet tropical forests.

Citación:

Vela Alvarado J.W., M. Rivas, V. Fernández \& M. Clavo Peralta. 2017. Mamíferos y aves silvestres usados por los pobladores de la cuenca del río Abujao (Ucayali, Perú). Revista peruana de biología 24(3): 263 - 272 (octubre 2017). doi: http://dx.doi.org/10.15381/rpb.v24i3.13907

Fuentes de financiamiento: El presente trabajo se realizó gracias a financiamiento de la Universidad Nacional de Ucayali a través del Fondo de Compensación del Gas de Camisea - Fondos FOCAM, en el marco del "Proyecto Abujao".

Permisos de colecta: La información colectada en el presente estudio contó con el consentimiento previo informado en los centros poblados de cuenca del río Abujao, como consta en actas.
Información sobre los autores:

JWVA, VFD, MCP: elaboración y diseño del estudio; JWVA, VFD: desarrollo de la base de datos, resumen de la información; MRS participó en la elaboración de la encuesta; organizó la logística de salida de campo; recolección de información de campo, resumen de la información e identificación taxonómica de las especies y nombres comunes; MCP. identificación taxonómica y nombres comunes de las especies; apoyo logístico en la salida de campo. JWVA: elaboración del manuscrito. Todos los autores revisaron y aprobaron el manuscrito.

Los autores no incurren en conflictos de intereses.

$\begin{array}{ll}\text { Presentado: } & 12 / 04 / 2017 \\ \text { Aceptado: } & 03 / 08 / 2017\end{array}$

Publicado online: $28 / 10 / 2017$ 


\section{Introducción}

Gran parte de las comunidades humanas habitantes en el Neotrópico, hacen uso de los ecosistemas y de sus componentes de diferentes maneras. En el caso de la fauna silvestre, existen diferentes modalidades de uso de especies, las cuales reflejan diferencias locales, sociales, económicas, culturales (Ojasti 1993).

El aprovechamiento de mamíferos y aves silvestres en la Amazonia es realizado por los pueblos indígenas, comunidades campesinas, colonos, agricultores, guarniciones militares, mineros, petroleros, turistas y la población urbana. Los usuarios indígenas y colonos de la fauna silvestre no son dos grupos completamente distintos; sin embargo, el uso que dan a la fauna silvestre los colonos difiere del aprovechamiento del recurso de parte de los indígenas (Bisbal 1994, Ojasti 1993, Redford \& Robinson 1987).

Por los diferentes tipos de uso, la contribución de la fauna al desarrollo económico y al bienestar social puede dividirse en dos categorías: Aportes directos en alimentos, carne y huevos; cueros y pieles; animales vivos para zoológicos e investigaciones biomédicas; colorantes, medicinas, productos para la magia; como adornos, plumas y cornamentas; animales taxidermizados para escuelas y universidades. Aportes indirectos a través de la caza deportiva, turismo y recreación, educación, usos de tierras marginales, promoción de comunidades rurales, la industria y el comercio (Álvarez 2007).

En la Amazonía se han realizado numerosos estudios para evaluar la importancia de las aves y mamíferos, así en la Reserva Nacional Pacaya-Samiria, cuencas de los ríos Ucayali y canal del Puinahua, en donde propusieron las bases para el manejo de las poblaciones de aves desde una óptica de conservación basada en el uso sostenible; determinándose la importancia relativa de las distintas especies de aves silvestres en los siguientes aspectos: caza de subsistencia, recolección de huevos, comercio de mascotas y otros usos como ritual, ornamental y turístico; al menos 47 especies de aves silvestres fueron aprovechados como alimentos destacando por su importancia el paujil (Mitu tuberosa), el sachapato (Cairina moschata), la panguana (Crypturellus undulatus), el cushuri (Phalacrocorax olivaceus) y la sharara (Anhinga anhinga). Otro rubro de importancia fue la captura y venta de aves para mascotas, y también como atractivos turísticos (González 1999).

En la zona del bajo Nanay, cerca de la ciudad de Iquitos, un estudio mostró que más del $70 \%$ de los ingresos económicos de las familias provenían de la extracción y comercialización de recursos naturales silvestres, especialmente madera, hojas de palmera para techado, pescado y fauna silvestre (Pyhälä 2003). En zonas más distantes de la ciudad este porcentaje se eleva hasta el 80 y 90\% de los ingresos económicos (IIAP 2004).

Para Álvarez (2007) "En la Amazonía, el respeto por la naturaleza y el cuidado de los recursos naturales no son un asunto romántico, sino una estrategia de supervivencia de la población rural: vive de los recursos naturales renovables, fauna y flora, $y$ cualquier mal manejo o sobreexplotación repercutirá directamente en su desarrollo futuro".

En la cuenca del río Abujao los centros poblados asentados en su área de influencia, hacen uso de aves y mamíferos silvestres para satisfacer sus diversas necesidades sin contar con medios de seguimiento y control sobre la frecuencia del aprovechamiento de estos recursos. Con el crecimiento de la población humana, en la cuenca del río Abujao está disminuyendo la biodiversidad y el conocimiento sobre su uso tradicional de las comunidades. El presente estudio fue realizado a través de encuestas estructuradas a informantes claves de 16 centros poblados de comunidades Ashéninkas, mestizas y Shipibo-Conibos, con el objetivo de monitorear el conocimiento del uso tradicional de especies de aves y mamíferos en la cuenca del río Abujao, incluida la determinación del valor de cada especie; la que debe servir como buena base de datos para investigaciones siguientes.

\section{Material y métodos}

El desarrollo de la fase de campo fue de octubre del 2008 a octubre del 2009 y actualizado al 2012, en la cuenca del río Abujao, región Ucayali, Amazonia peruana, frontera con Brasil, que forma parte de la Sierra de Divisor (Fig. 1). La cuenca del río Abujao es una unidad geográfica que comprende un área de 349,262 ha, ubicada entre las coordenadas UTM 585191.28

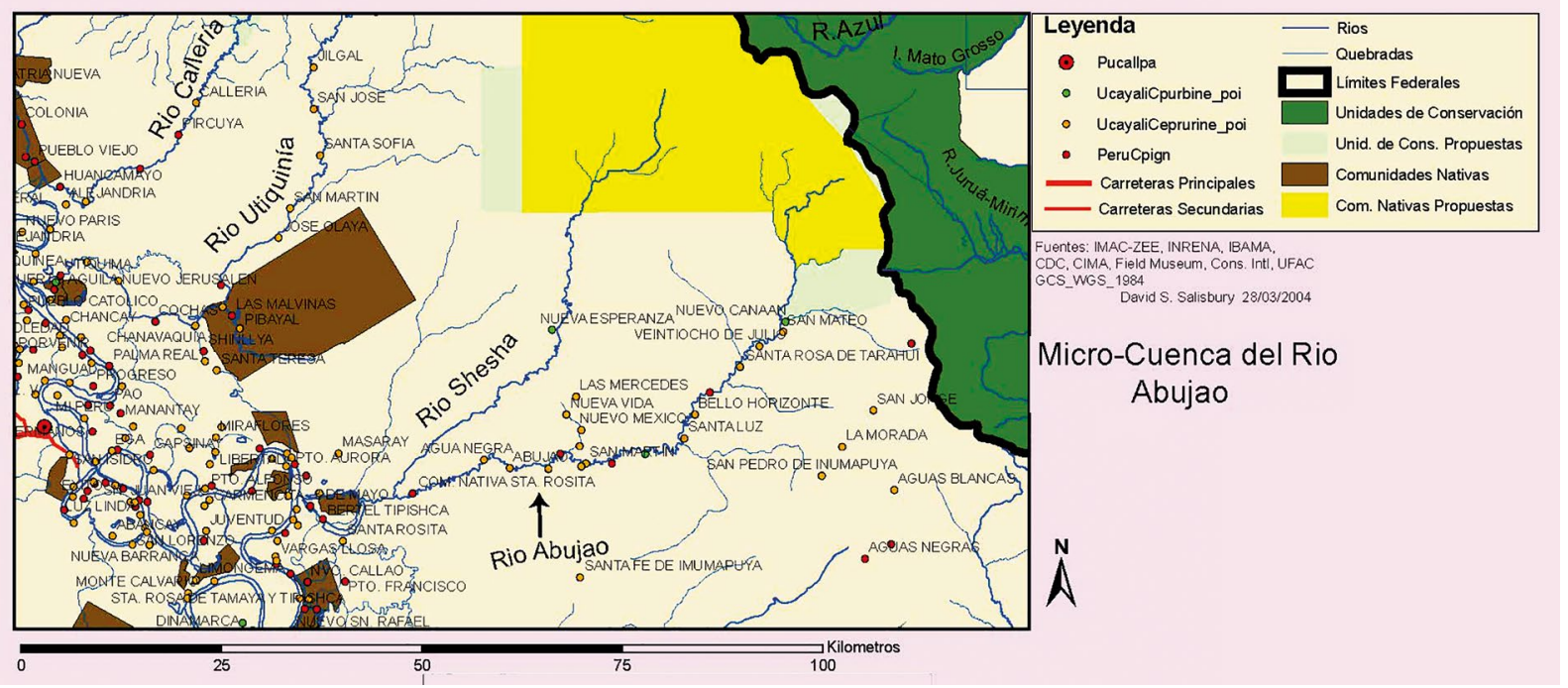

Figura 1. Cuenca del río Abujao 
E y 643971.86 E y $9062718.87 \mathrm{~N}$ y $9093962.03 \mathrm{~N}$ entre las cuencas de los ríos Utiquinía y Tamaya (CIFA 2009). Presenta un clima semi húmedo cálido a súper húmedo cálido. En la cuenca existen 16 pueblos de los cuales 14 son mestizos y 2 son indígenas, de éstos se escogieron 6 pueblos como lugares de muestreo debido a las condiciones que ofrecían para el estudio (Tabla 1). Se incluyeron tanto comunidades mestizas como indígenas, que tienen una significativa población en relación al resto, y además son equidistantes a lo largo de la cuenca, seleccionándose en la Cuenca Alta, Media y Baja.

Para obtener los datos, se aplicaron entrevistas estructuradas, observación directa y una guía ilustrada para la identificación de especies reconocidas y utilizadas por la población. Las encuestas se aplicaron al $25 \%$ del total de las familias en cada comunidad. Los usos se agruparon en las siguientes categorías: uso alimenticio, como mascota, mágico y ritual, medicinal, artesanal y ornato, comercial y otros usos que no están incluidos dentro de los anteriores. Toda la información fue guardada y grabada como audio. Para asegurar la calidad sobre el reconocimiento de las especies se alcanzó al informante un catálogo con las imágenes de las especies más frecuentes en la zona.

Análisis de datos.- Las variables independientes fueron determinadas por observación del investigador: centros poblados nivel de desarrollo de la población, educación, actividades humanas (Tabla 1).

Las variables dependientes fueron las especies de animales que se encuentran en la zona investigada. Número de especies cuyo conocimiento sobre su uso forma parte de la cultura de los grupos humanos estudiados.
Con los datos obtenidos de las entrevistas se elaboró una tabla con la clasificación taxonómica. Para el análisis e interpretación de los datos obtenidos se organizaron y caracterizaron sistemáticamente utilizándose la estadística descriptiva e inferencial. Para conocer el valor de uso de las especies se utilizaron los siguientes índices (Phillips \& Gentry 1993):

- Valor de diversidad de uso que tiene cada especie (VDE).- Con la finalidad de conocer la importancia de cada especie animal como especie útil para los pobladores de la cuenca del Abujao. (VDE - valor de diversidad de uso de cada especie; $n U$ - número de usos de una especie; $n T U$ - total de usos registrados.)

$$
\mathrm{VDE}=\mathbf{n U} / \mathrm{Ntu}
$$

- Valor de diversidad para cada uso (VDU).- Con la finalidad de conocer la importancia de cada uso para los pobladores de la cuenca del Abujao. (VDU - Valor de diversidad para cada uso; $n E$ - Número de especies por uso; $n T E$ - Total de especies registradas.)

$$
\mathrm{VDU}=\mathbf{n E} / \mathbf{n T E}
$$

- Valor de diversidad de uso de partes o productos de cada animal (VDP).- Este cálculo se realizará con la finalidad de analizar la importancia de cada especie animal a partir de la cantidad de partes o productos utilizados. (VDP - Valor de diversidad de uso de partes o productos de cada animal; $n P U$ - Número de partes usadas de cada animal; $n T P$ : Total de partes registradas.)

$\mathrm{VDP}=\mathbf{n P U} / \mathbf{n T P}$

\begin{tabular}{|c|c|c|c|c|}
\hline $\begin{array}{l}\text { Centro } \\
\text { Poblado }\end{array}$ & Descripción de la zona & Ubicación geográfica & Vivientes & Actividades humanas \\
\hline San Mateo & $\begin{array}{l}\text { Alto Abujao - terrenos } \\
\text { adyacentes a la zona } \\
\text { fronteriza con Brasil, distrito } \\
\text { de Callería, región Ucayali }\end{array}$ & $\begin{array}{l}\text { UTM 643971.86E } \\
\text { 9093962.03N; a } 181.9 \mathrm{~km} \\
\text { de Pucallpa }\end{array}$ & $\begin{array}{l}\text { Comunidad nativa - } \\
\text { etnia Asháninka }\end{array}$ & $\begin{array}{l}\text { Agricultores, colectores, } \\
\text { cazadores, pescadores }\end{array}$ \\
\hline 28 de Julio & $\begin{array}{l}\text { Cuenca media del río Abujao } \\
\text { - colinas onduladas y algo } \\
\text { escarpadas }\end{array}$ & $\begin{array}{l}\text { UTM 624732.45E } \\
9070935.25 \mathrm{~N} ; 171 \mathrm{msnm} ; \mathrm{a} \\
120 \mathrm{~km} \text { de Pucallpa }\end{array}$ & $\begin{array}{l}\text { Población diversa } \\
\text { mayormente emigrantes } \\
\text { de la zona andina y de } \\
\text { Brasil y Bolivia. }\end{array}$ & $\begin{array}{l}\text { Pocos agricultores, } \\
\text { mayormente minería }\end{array}$ \\
\hline $\begin{array}{l}24 \text { de } \\
\text { Setiembre }\end{array}$ & $\begin{array}{l}\text { Cuenca media del río Abujao } \\
\text { - colinas onduladas y algo } \\
\text { escarpadas }\end{array}$ & $\begin{array}{l}\text { UTM 620200.03E } \\
\text { 9069357.06N; } 171 \text { msnm; } \\
111.52 \text { km de Pucallpa }\end{array}$ & & $\begin{array}{l}\text { agricultura a pequeña escala, } \\
\text { caza, extracción maderera, } \\
\text { crianza de animales menores }\end{array}$ \\
\hline Santa Luz & $\begin{array}{l}\text { Cuenca media del río Abujao } \\
\text { - terrazas aluviales no } \\
\text { inundables }\end{array}$ & $\begin{array}{l}\text { UTM 603330.10E } \\
\text { 9067539.29N, } 154 \text { msnm, } \\
78.60 \text { km de Pucallpa }\end{array}$ & Mestizos & $\begin{array}{l}\text { Agricultura medio } \\
\text { desarrollada, crianza } \\
\text { de animales, extracción } \\
\text { maderera, poco de minería }\end{array}$ \\
\hline Abujao & $\begin{array}{l}\text { Parte baja de la cuenca } \\
\text { del río Abujao - terrazas } \\
\text { aluviales no inundables }\end{array}$ & $\begin{array}{l}\text { UTM 592508.97E } \\
\text { 9064619.67N, } 149 \text { msnm, } \\
\text { 56.90 Km de Pucallpa }\end{array}$ & Mestizos & $\begin{array}{l}\text { Agricultura, comercio, } \\
\text { ganadería, caza, extracción } \\
\text { maderera, crianza de animales } \\
\text { menores. }\end{array}$ \\
\hline $\begin{array}{l}\text { Santa } \\
\text { Rosa de la } \\
\text { Tipishca } \\
\text { del Tamaya }\end{array}$ & $\begin{array}{l}\text { parte baja de la cuenca del } \\
\text { río Abujao - terrazas bajas }\end{array}$ & $\begin{array}{l}\text { UTM 585191.28E } \\
9062718.87 N, 144 \text { msnm, a } \\
43.60 \text { km de Pucallpa }\end{array}$ & $\begin{array}{l}\text { Comunidad nativa de } \\
\text { Shipibo-Conibo (grupo } \\
\text { etnolinguístico Pano) }\end{array}$ & $\begin{array}{l}\text { Agricultura, pesca, artesanía, } \\
\text { crianza de animales menores, } \\
\text { tratamiento de enfermedades } \\
\text { con plantas medicinales. }\end{array}$ \\
\hline
\end{tabular}

Tabla1. Variables independientes - características de los centro poblados en la cuenca del río Abujao 


\section{Resultados}

Los pobladores de la cuenca del río Abujao reconocieron como recurso faunístico silvestre utilizados un total de 62 especies de las cuales 35 fueron mamíferos y 27 aves. En mamíferos se identificaron 7 órdenes, donde destacan Artiodactyla, Primates y Rodentenia; en relación a las aves, se encontraron un total de 12 órdenes donde destacan Psittaciformes, Galliformes y Tinamiformes (Tablas 3 y 4). Con respecto al número de especies utilizada por centro poblado se observa la mayor cantidad de especies registradas en la comunidad Nativa Shipibo - Conibo de Santa Rosa de Tamaya Tipishca, con un total de 47 especies y el menor número de especies en el centro poblado 24 de setiembre donde se registraron solamente 26 especies (Fig. 2).

Evaluación de uso de las especies silvestres.- En los centros poblados de la cuenca del río Abujao fueron determinados seis tipos de uso de la fauna silvestre (mamíferos y aves): uso alimentario, como mascota, por razones mágicas o tradicionales, medicinal, artesanal y uso comercial (Tabla 2).

Uso alimenticio.- Se identificaron 33 especies de mamíferos y 22 especies de aves con uso alimenticio.

Para el grupo de mamíferos se encontraron en total de 20 familias entre ellas las más representativas resultaron: Tayassuidae, Agoutidae, Cervidae. Para aves fueron registrados en total de 13 familias destacando entre ellas: Cracidae, Psittacidae y Tinamidae. Entre los factores que influyen sobre el consumo de las especies encontramos que la carne de la especie no es apreciable para el consumo, que existe abundancia de especies de mayor preferencia y a restricciones por cuestiones culturales, específicamente en el caso del "huapo colorado" (Cacajao calvus). Con respecto al número de especies consumidas por centro poblado se observa que en el centro Abujao y la Comunidad Nativa Santa Rosa presentaron similitudes en la cantidad de especies utilizadas siendo 38 las especies registradas para cada una, mientras que en otros centros poblados disminuye este número tan solo a 20 (Fig. 3 y Tablas 3 y 4).

Mascota.- Especies utilizadas por fines personales como mascota fueron representadas por 20 especies de mamíferos y 20 especies de aves. Se encontraron 12 familias de mamíferos siendo las de mayor importancia Atelidae, Callitrichidae, Cebidae. También fueron registradas 7 familias de aves destacando por su mayor uso como mascota las familias Psittacidae y Cracidae. Para el uso como mascota en general los más preferidos fueron los primates "choro" Lagothrix lagothricha y el "pichico barba blanca" Sanguinus mystax. Esto demuestra que hay una gran preferencia por la crianza de primates como mascotas por su apariencia humana y bonachona además de ser especies muy fáciles de domesticar y de gran adaptabilidad a la convivencia

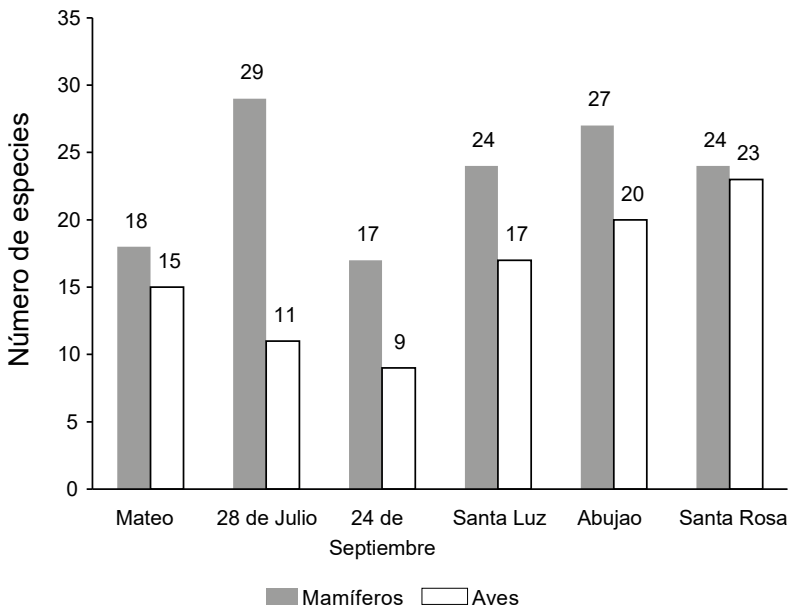

Figura 2. Números de especies registrados por centro poblado.

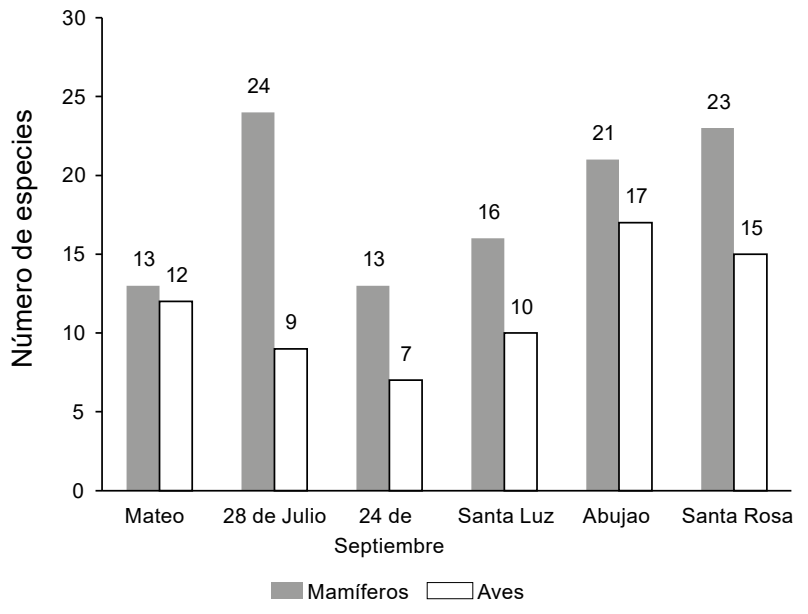

Figura 3. Número de especies de uso alimenticio por centro poblado

Tabla 2. Categorías de uso de los mamíferos y aves silvestres por los vivientes en la cuenca del río Abujao

\begin{tabular}{|c|c|}
\hline Categoría & Especificación \\
\hline Alimentario & $\begin{array}{l}\text { Se refiere al uso para el autoconsumo, donde la población captura o caza alguno de estos animales para sufragar sus } \\
\text { necesidades de alimentación }\end{array}$ \\
\hline Mascota & $\begin{array}{l}\text { Es la relación afectiva o de posesión que pueda tener el propietario de un animal con éste, en la cual el fin no es } \\
\text { comercializarlo o consumirlo. }\end{array}$ \\
\hline Mágico y ritual & $\begin{array}{l}\text { Es la utilización de animales dentro de la relación que tienen unas especies con mitos, tradiciones o leyendas en las } \\
\text { poblaciones. }\end{array}$ \\
\hline Medicinal & Este tipo de uso se refiere a las propiedades curativas con las que son relacionados los animales en las poblaciones rurales. \\
\hline $\begin{array}{l}\text { Artesanal y } \\
\text { ornato }\end{array}$ & $\begin{array}{l}\text { Este uso se da cuando los productos derivados de la fauna son usados como ornato para resaltar o aumentar el grado } \\
\text { estético o importancia de alguna persona, lugar u objeto. }\end{array}$ \\
\hline Comercial & Se refiere al aporte económico de la fauna silvestre tras la venta de especies o de algún subproducto de éstas. \\
\hline Otros usos & Se considerará algún otro uso que no esté incluido dentro de los anteriores. \\
\hline
\end{tabular}


Tabla 3. Familias y especies de la fauna silvestre clase mamíferos, usadas por pobladores de la cuenca del Abujao. Departamento de Ucayali, Perú, 2009 - 2012

\begin{tabular}{|c|c|c|c|c|c|c|c|}
\hline \multirow{2}{*}{ Orden } & \multirow{2}{*}{ Familia/Nombre científico } & \multicolumn{3}{|c|}{ Nombre Común } & \multirow{2}{*}{ Usos* } & \multirow{2}{*}{ VDE } & \multirow{2}{*}{ VDP } \\
\hline & & Español & Shipibo Conibo & Asheninka & & & \\
\hline \multirow[t]{2}{*}{ Marsupialis } & Didelphidae & & & & & & \\
\hline & Didelphys marsupialis & Intuto, zorro & Maxo & Zagari / Sari & $\mathrm{Me}$ & 0.167 & 0.038 \\
\hline \multirow[t]{5}{*}{ Xenarthros } & Dasyodidae & & & & & & \\
\hline & Dasypus novemcintus & Carachupa & Yawish & Etzi & $\mathrm{A}, \mathrm{Me}, \mathrm{Ar}$ & 0.667 & 0.077 \\
\hline & Priodontes maximus & Yangunturo & & Kintero & $\mathrm{A}, \mathrm{Me}$ & 0.333 & 0.154 \\
\hline & Myrmecophagidae & & & & & & \\
\hline & Mymecophaga tridactyla & Oso hormiguero & Xae & Shaani / Sha & $\mathrm{A}, \mathrm{Me}$ & 0.333 & 0.115 \\
\hline \multirow[t]{18}{*}{ Primates } & Callitrichidae & & & & & & \\
\hline & Saguinus mystax & $\begin{array}{l}\text { Pichico barba } \\
\text { blanca }\end{array}$ & Shipi & Tsipi & $\mathrm{A}, \mathrm{Ma}, \mathrm{Me}$ & 0.500 & 0.154 \\
\hline & Saguinus fuscicollis & Pichico común & Shipi & Tsipi & $\mathrm{A}, \mathrm{Ma}, \mathrm{Me}$ & 0.500 & 0.154 \\
\hline & Callimico goeldii & Supay Pichico & Shipi & Tsipi & & 0.167 & 0.115 \\
\hline & Cebidae & & & & & & \\
\hline & Saimiri sp. & Huasa & Wasa & Tsiyeri & $\mathrm{A}, \mathrm{Ma}, \mathrm{Me}$ & 0.667 & 0.115 \\
\hline & Cebus apella & $\begin{array}{l}\text { Machín negro, } \\
\text { mono negro }\end{array}$ & Wiso shino & Shitoni & $\mathrm{A}, \mathrm{Ma}, \mathrm{Me}, \mathrm{A}$ & 0.833 & 0.231 \\
\hline & Cebus albifrons & $\begin{array}{l}\text { Machin blanco, } \\
\text { mono blanco }\end{array}$ & Joxo shino & Koshiri & A & 0.333 & 0.077 \\
\hline & Aotidae & & & & & & \\
\hline & Aotus nigricepts & Musmuqui & Riro & Pitoni & $\mathrm{A}, \mathrm{Ma}$ & 0.333 & 0.115 \\
\hline & Piteciidae & & & & & & \\
\hline & Callicebus cupreus & Tocón & Roka roka & Tookori & & 0.167 & 0.077 \\
\hline & Cacajao calvus & Huapo colorado & Jopshin wapo & $\begin{array}{l}\text { Geroniaichokari / } \\
\text { Gero }\end{array}$ & A & 0.167 & 0.077 \\
\hline & Pithecia monachus & Huapo negro & Wiso wapo & Geroni & $\mathrm{A}, \mathrm{Ma}$ & 0.500 & 0.154 \\
\hline & Atelidae & & & & & & \\
\hline & Alouatta seniculus & Coto & Ro & Shenontzi / Taniri & $\mathrm{A}, \mathrm{Ma}, \mathrm{Me}, \mathrm{Ar}, \mathrm{C}$ & 0.833 & 0.231 \\
\hline & Ateles paniscus (Chamek=) & Maquisapa & Isso & Thowero & $\mathrm{A}, \mathrm{Ma}, \mathrm{C}$ & 0.500 & 0.154 \\
\hline & Lagothrix lagotricha & Choro & Iso & Osheto & $\mathrm{A}, \mathrm{Ma}, \mathrm{Me}, \mathrm{C}$ & 0.667 & 0.231 \\
\hline \multirow[t]{8}{*}{ Carnivoros } & Felidae & & & & & & \\
\hline & Leopardus pardalis & Trigrillo & Ino & Mathonthori & A, Ar, C & 0.500 & 0.154 \\
\hline & Panthera onca & Otorongo, tigre & Ino & Kashekari / Manitzy & $\mathrm{A}, \mathrm{Me}, \mathrm{Ar}, \mathrm{C}$ & 0.667 & 0.115 \\
\hline & Mustelidae & & & & & & \\
\hline & Eira barbara & Manco & Boca & owaatzi & A & 0.167 & 0.038 \\
\hline & Procyonidae & & & & & & \\
\hline & Nasua nasua & Achuni & Shishi & Kapeshi & $\mathrm{A}, \mathrm{Ma}, \mathrm{Me}$ & 0.667 & 0.192 \\
\hline & Potos flavus & Chosna & & Pito & A & 0.167 & 0.038 \\
\hline \multirow[t]{5}{*}{ Perissodactyla } & Tapiridae & & & & & & \\
\hline & Tapirus terrestres & Sachavaca & Awa & Kemari & $\mathrm{A}, \mathrm{Ma}, \mathrm{Me}, \mathrm{C}$ & 0.667 & 0.269 \\
\hline & Tayassuidae & & & & & & \\
\hline & Pecari tajacu & Sajino & Jono & Kitairiki & $\mathrm{A}, \mathrm{Ma}, \mathrm{Me}, \mathrm{Ar}, \mathrm{C}$. & 0.833 & 0.231 \\
\hline & Tayassu pecari & Huangana & Yawa & Shintori & $\mathrm{A}, \mathrm{Ma}, \mathrm{Ar}, \mathrm{C}$ & 0.667 & 0.192 \\
\hline \multirow[t]{3}{*}{ Artiodactyla } & Cervidae & & & & & & \\
\hline & Mazama americana & Venado colorado & Chaxo & Ponawari & $\mathrm{A}, \mathrm{Ma}, \mathrm{Me}, \mathrm{Ar}, \mathrm{C}$ & 0.833 & 0.308 \\
\hline & Mazama gouzoubira & Venado cenizo & Chaxo & Kairishi & $\mathrm{A}, \mathrm{Ma}, \mathrm{Me}, \mathrm{Ar}, \mathrm{C}$ & 0.833 & 0.269 \\
\hline \multirow[t]{15}{*}{ Rodentia } & Sciuridae & & & & & & \\
\hline & Sciurus sp. & Ardilla, waiwasho & Capa & Tsiyeri & $\mathrm{A}, \mathrm{Ar}$ & 0.333 & 0.077 \\
\hline & Erethizontidae & & & & & & \\
\hline & Coendu bicolor & $\begin{array}{l}\text { Casha cushillo, } \\
\text { Puerco espín, erizo }\end{array}$ & Isa & Tontori & $\mathrm{A}, \mathrm{Me}$ & 0.333 & 0.077 \\
\hline & Hydrochaeridae & & & & & & \\
\hline & Hydrochaeris hydrochaeris & Ronsoco & Amen & Oveto / Ipetzi & $\mathrm{A}, \mathrm{Ma}, \mathrm{Me}, \mathrm{Ar}$ & 0.833 & 0.231 \\
\hline & Dasyproctidae & & & & & & \\
\hline & Dasyprocta fuliginosa & Añuje & Mari & Sharo / Shawo & $\mathrm{A}, \mathrm{Ma}, \mathrm{C}$ & 0.500 & 0.154 \\
\hline & Myoprocta pratti & Punchana & & & $\mathrm{A}, \mathrm{Ma}$ & 0.333 & 0.115 \\
\hline & Agoutidae & & & & & & \\
\hline & Agouti paca & Majás, Picuro & Ano & Samani & $\mathrm{A}, \mathrm{Me}, \mathrm{Ar}, \mathrm{C}$ & 0.833 & 0.231 \\
\hline & Dactylomys & & & & & & \\
\hline & Dactylomys sp. & Cono cono & & & A & 0.167 & 0.038 \\
\hline & Echimyidae & & & & & & \\
\hline & Proechimys & Sachacuy & Хoya & Onkiro & $\mathrm{A}$ & 0.167 & 0.038 \\
\hline
\end{tabular}


Tabla 4. Familias y especies de la fauna silvestre clase aves, usadas por pobladores de la cuenca del río Abujao. Departamento de Ucayali, Perú, 2009-2012

\begin{tabular}{|c|c|c|c|c|c|c|c|}
\hline \multirow{2}{*}{ Orden/ Familia } & \multirow{2}{*}{ Nombre científico } & \multicolumn{3}{|c|}{ Nombre Común } & \multirow{2}{*}{ Usos* } & \multirow{2}{*}{ VDE } & \multirow{2}{*}{ VDP } \\
\hline & & Español & Shipibo Conibo & Asheninka & & & \\
\hline \multicolumn{8}{|l|}{ Anseriformes } \\
\hline Anhimidae & Anhima cornuta & Camungo & Abo koma & Amikomotzi & Ma, & & \\
\hline Anatidae & Cairina moschata & $\begin{array}{l}\text { Sacha pato, pato de } \\
\text { monte, yacu pato }\end{array}$ & Jenen ponpon & Pantyo & A & 0.167 & 0.038 \\
\hline \multicolumn{8}{|l|}{ Charadriiformes } \\
\hline Jacanidae & Jacana jacana & Tuqui tuqui & Nepaxtaki & Kowacherityonki & $\mathrm{A}, \mathrm{Me}$ & 0.333 & 0.077 \\
\hline \multirow{3}{*}{ Ardeidae } & Egretta thula & Garza blanca chica & Joxo manxan & Thompori & $\mathrm{A}, \mathrm{Ma}, \mathrm{Ar}, \mathrm{C}$ & 0.667 & 0.192 \\
\hline & Tigrisoma lineatun & Puma garza & Jaka & Sananti & $\mathrm{A}$ & 0.167 & 0.115 \\
\hline & Ardea cocoi & $\begin{array}{l}\text { Garza ceniza, } \\
\text { Uchpa garza }\end{array}$ & Piti manxan & & A & 0.167 & 0.115 \\
\hline \multicolumn{8}{|l|}{ Ciconiiformes } \\
\hline $\begin{array}{l}\text { Ciconiidae } \\
\text { Columbiformes }\end{array}$ & Mycteria americana & Manshaco & Manxan teo & & A & 0.167 & 0.038 \\
\hline \multicolumn{8}{|l|}{ Falconiformes } \\
\hline \multirow{2}{*}{ Accipitridae } & Buteo magnirostris & Aguilucho & & Merotzi / Meyotzi & $\mathrm{C}$ & 0.167 & 0.038 \\
\hline & Geranospiza caerulescens & Gavilán de pata roja & Tete & Pakitha & $\mathrm{R}$ & 0.167 & 0.077 \\
\hline Falconidae & Daptrius americanus & Atatao & Atatao & Aarón / Awo & C & 0.167 & 0.038 \\
\hline \multicolumn{8}{|l|}{ Galliformes } \\
\hline \multirow{4}{*}{ Cracidae } & Ortalis guttata & Manacaraco & Jene karaka & Maratzi / Matiz & A, Ma, & 0.333 & 0.154 \\
\hline & Mitu mitu & Paujil & Jasin & Thamari & $\mathrm{A}, \mathrm{Me}$ & 0.500 & 0.192 \\
\hline & Penelope jacquacu & Pucacunga & Kebo & Sankatzi & $\mathrm{A}, \mathrm{Ma}$, & 0.500 & 0.154 \\
\hline & Pipile pipile & Pava de monte & Koxo & Chooni / Tsioni & A & 0.167 & 0.077 \\
\hline \multicolumn{8}{|l|}{ Gruiformes } \\
\hline Eurypygidae & Eurypyga helias & Tanrrilla & & Shorintzi & $\mathrm{R}$ & 0.167 & 0.038 \\
\hline Psophiidae & Psophia crepitans & Trompetero & Nea & Chakami & A & 0.167 & 0.038 \\
\hline \multicolumn{8}{|l|}{ Passeriformes } \\
\hline Icteridae & Cacicus cela & Paucar & Chana & Thoori & A, Ar, C & 0.500 & 0.077 \\
\hline \multicolumn{8}{|l|}{ Pelecaniformes } \\
\hline Anhingidae & Anhinga anhinga & Sharara, Anhinga & Xara ra & Tsinke & A & 0.333 & 0.115 \\
\hline Phalacrocoracidae & Phalacrocórax olivaceus & $\begin{array}{l}\text { Coromorán, } \\
\text { cushuri }\end{array}$ & Koshori & Katari & $\mathrm{A}, \mathrm{Ma}$, & $\mathrm{x}$ & 0.115 \\
\hline $\begin{array}{l}\text { Piciformes } \\
\text { Ramphastidae } \\
\text { Psittaciformes }\end{array}$ & Ramphastos cuvieri & Tucán & Xoke & Opempe & A, Ar, C & 0.500 & 0.077 \\
\hline \multirow{4}{*}{ Psittacidae } & Ara chloroptera & Guacamayo & Xawan & Sahuaro & $\mathrm{A}, \mathrm{Ma}, \mathrm{Ar}, \mathrm{C}$ & 0.667 & 0.115 \\
\hline & Ara macao & Guacamayo rojo & Joshin xawan & Sari & $\mathrm{A}, \mathrm{Ma}, \mathrm{Ar}, \mathrm{C}$ & 0.667 & 0.115 \\
\hline & Brotogeris sp. & Pihuicho & Pitso & Chorito / Chokiyo & $\mathrm{A}, \mathrm{Ma}$ & 0.333 & 0.077 \\
\hline & Amazona festiva & Loro hablador & Bawa & $\begin{array}{l}\text { Santero / Ezotzi / } \\
\text { Kentaro }\end{array}$ & $\mathrm{A}, \mathrm{Ma}, \mathrm{Ar}, \mathrm{C}$ & 0.667 & 0.115 \\
\hline \multicolumn{8}{|l|}{ Tinamiformes } \\
\hline Tinamidae & Tinamos major & Perdiz & Koman ewa & Shunkiri & A, Ma, & 0.500 & 0.154 \\
\hline & Crypturellus undulatus & Panguana & Koma & Maakokawa & $\mathrm{A}, \mathrm{Ma}$, & 0.500 & 0.154 \\
\hline
\end{tabular}

$\mathrm{A}=$ alimentario, $\mathrm{Ma}=$ mascota, $\mathrm{Me}=$ medicinal, $\mathrm{R}=$ ritual, $\mathrm{C}=$ comercial, $\mathrm{Ar}=$ artesanal

$\mathrm{VDE}=$ Valor de Diversidad de Uso de la espécie; $\mathrm{VDP}=$ Valor de Diversidad de las partes de la especie

con humanos. De las aves los más preferidos son "pihuicho" Brotogeris sp., "loro" Amazona festiva y "guacamayo" Ara sp. Como en el caso del uso alimentario, también en uso de mascotas figura en el primer puesto el centro poblado Abujao con 25 especies en total, seguido con los de mas centros poblados con números menores disminuyendo hasta 9 o 10. También se manifiesta intenciones para la cría de ungulados y roedores como el "ronsoco" Hydrochaeris hydrochaeris por la facilidad de ser domesticado y su docilidad. Los principales motivos para la cría de aves como mascota son la vistosidad y colorido de su plumaje, la peculiaridad de sus cantos, el poder pronunciar algunas palabras o imitar sonidos, el bullicio y la compañía. En la Comunidad Nativa Santa Rosa también se ha registrado el uso del "camungo" Anhima corneta, que con sus sonidos anuncia al morador que se acerca alguien desconocido a la casa (Fig. 4 y Tablas 3 y 4 )

Uso mágico y ritual.- En la categoría del uso mágico y ritual se registraron solamente 4 especies de aves, donde la mayor representatividad tuvo la "tanrrilla" Eurypyga helias (Eurypygidae), seguido del "tatatao" Daptrius americanus (Falconiformes), 
"paloma torcaza" Columba plúmbea (Columbidae) y el "gavilán de pata roja" Geranospiza caerulescens (Accipitridae). Se ha registrado que el uso más frecuente estas especies se dan en las preparaciones de "pusanga" (hechizo de amor) a excepción de la "tanrrilla" que también es utilizada como amuleto para la cacería y la pesca. La Figura 5 (Tablas 3 y 4) presenta el uso de las especies de uso mágico y ritual por centros poblados. La lo largo de la cuenca fue observada la presencia de varias iglesias cristianas: católica, adventista, evangélica e israelitas, quienes según sus convicciones tienden a prohibir ciertas prácticas tradicionales. Es posible que esto haya influido sobre el número de especies registradas para el Uso Mágico Ritual y también posiblemente para el Uso Medicinal.

Uso medicinal.- Es interesante que se identificaran 19 especies de mamíferos y solo 2 especies de aves para usos medicinales. Para el grupo de mamíferos se encontraron 14 familias, entre ellas las más representativas resultaron Cervidae, Tapiridae y Cebidae. Especies con mayor uso para el propósito medicinal fueron: "sachavaca" (Tapirus terrestres) que cura 4 enfermedades así mismo como el venado colorado (Mazama americana) y Cashacuchillo (Coendu bicolor) es utilizado para curar 5 enfermedades o dolencias diferentes. Entre las aves el uso medicinal tiene Paujil (Mitu mitu) de la familia Cracidae y "tuqui tuqui" (Jacana jacana) de la familia Jacanidae.

Para la medicina tradicional se registraron 13 partes de animales en las cuales destacaron mas: la grasa que es extraída de 11 especies y es utilizada para diversas dolencias, el uso de testículos y miembro viril con 7 especies, que es utilizado para tratar y prevenir problemas de impotencia y también se registró el uso de la hiel en 6 especies como antiofídico. Es importante destacar aquí, que no existe unanimidad entre las personas que curan, en cuanto a los efectos terapéuticos y modos de administración de las medicinas naturales. Sin embargo, estas diferencias no se perciben como contradicciones, sino como estilos o formas diferentes de curar, por lo que no afectan su credibilidad. En la cuenca del río Abujao se reconoce que la principal enfermedad o dolencia tratada con mamíferos y aves silvestres es la impotencia, seguidamente de uso como antiofídico; también para el tratamiento de bronquios, asma y tosferina. En el centro poblado Abujao se registró la mayor cantidad de especies utilizadas con fines medicinales (13), en otros caseríos el numero fluctúa entre tres y seis especies y en San Mateo utilizan sola una especie (Fig. 6 y Tablas 3 y 4$)$.

Uso artesanal y ornamental.- Para el grupo de mamíferos se identificaron 8 familias de las cuales la mayor importancia tiene Tayassuidae, Cervidae y Felidae. Siendo las especies más reconocidas: "Venado colorado" Mazama americana, "sajino" Pecari tajacu, "Trigrillo" Leopardus pardalis, y "otorongo" Panthera onca. Para el grupo de aves se registraron 4 familias entre las que predomina la familia Psittasidae con el mayor número de especies: "Loro" Amazona festiva y "Guacamayos" Ara sp. También se reconoce cierta importancia a la familia Ardeidae que tiene a la "Garza blanca" Egretta thula como su especie más representativa. En varios productos ornamentales se mayormente reconocen cueros, cuernos, cráneos y dentaduras. En cuanto a las aves se usan las plumas. Se ha registrado que es una práctica común el decorar la casa colocándole algún animal o parte de este, entre ellas: osamentas, pieles, plumas, algún miembro, etc. La mayoría de los centros poblados esta utilizando plumas decorativas de los aves ornamentales como el loro (Amazona sp.) y guacamayo (Ara sp.), que son usadas para vestimenta, arcos y flechas, coronas y maracas. También destaca "venado colorado"

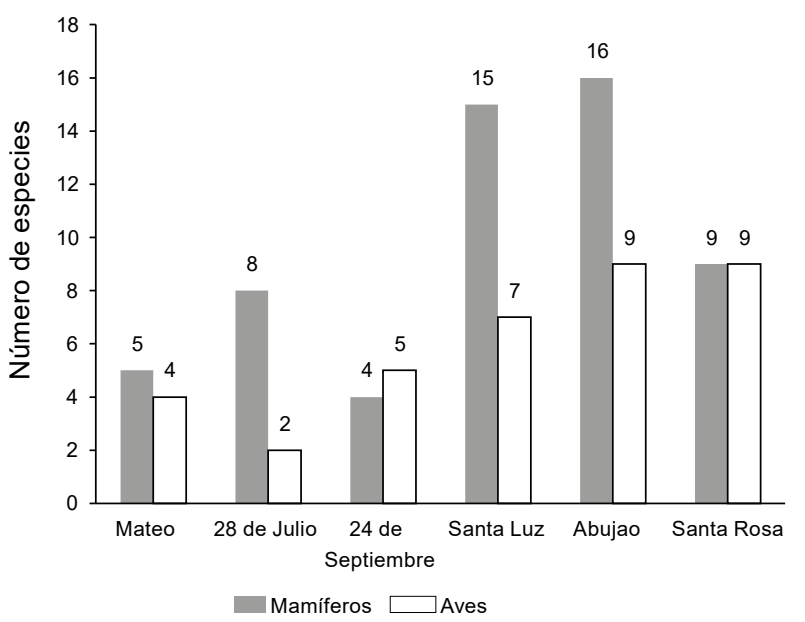

Figura 4. Número de especies de uso como mascota por centro poblado.

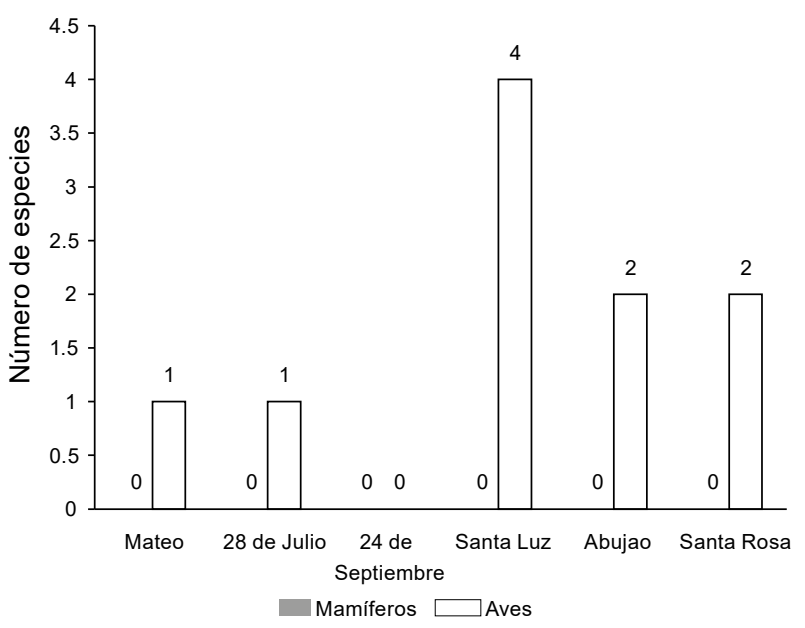

Figura 5. Número de especies de uso Mágico - Religioso por centro poblado.

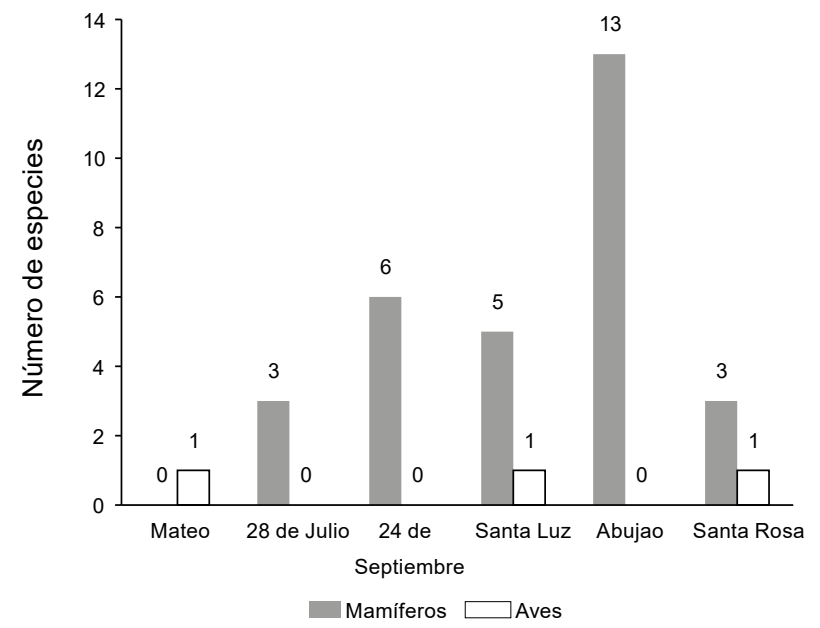

Figura 6. Número de especies de uso medicinal por centro poblado. 
Mazama americana, que es utilizada como adorno para la casa y para la elaboración de bombos y redoblantes.

Mayor uso de animales silvestres por los fines ornamentales fue registrada en la Comunidad nativa Santa Rosa (19 especies) en contrario menor uso representan los centros San Mateo y 24 de Setiembre (4 especies) (Tablas 3 y 4).

Uso comercial.- El uso comercial de los mamíferos y aves silvestres empieza con la extracción del bosque cazando, atrapando o colectando en estadías juveniles. La caza comercial de mamíferos y aves silvestres en la cuenca del río Abujao se practica en varias formas y escalas, de similar manera con lo reportado en otros ámbitos de la cuenca amazónica, como Ojasti (2000) quien describe que la caza comercial la puede realizar un cazador de subsistencia que vende las pieles de animales abatidos para el consumo doméstico o una campesina que cría pichones de perico con miras a su venta, pasando por el cazador de oficio o artesanal que opera por su cuenta, hasta la caza comercial organizada, donde el cazador es apenas un obrero de hacienda o empresa a cargo de la matanza y el procesamiento de las presas.

Para el uso comercial se identificaron 17 especies de mamíferos y 12 especies de aves. El grupo de mamíferos destacan Artiodactyla, Primates y Rodentia representados por 11 familias, representadas por Tayassuidae, Agoutidae y Atelidae. Para las aves fueron identificadas 8 familias, entre las que destaca ampliamente es Psittacidae y Cracidae. Se registró que entre los factores que influyen sobre el mayor o poco uso de estas especies para el comercio varía desde el poco interés para adquirir estas especies, la dificultad para capturarlas, la comercialización no justifica el costo por extracción, existe abundancia de especies de mayor preferencia (Tablas 3 y 4 ).

En la comercialización de productos oranemntales predomina la carne, cuero y plumas. Con mayores precios se comercializa "majás" Agouti paca (US \$2.5 kg, dólar americano). Luego "sajino" Tayassu tajacu, "Venado colorado" Mazama americana y "Huangana" Tayassu pecari Mientras en aves solo se registraron 6 especies poco significativas para el comercio: "perdiz" Tinamos major, "garza" Egretta thula y "Sharara" Anhinga anhinga de precio a US \$1.00. (1 US \$ es igual a S./ 2.9 nuevos soles).

La comercialización de la carne se inicia con la extracción desde el bosque realizada por los cazadores, quienes suelen vender a intermediarios y/o abastecer al mercado local, o también, venderla fuera de la cuenca. Los regatones a su vez también suelen abastecer al mercado local pero prefieren comprar en la cuenca y vender la carne a los mercados externos como San Juan, Mazaray y Pucallpa (distancia de 45 a $110 \mathrm{~km}$ ), donde tienen mayores utilidades. Se registró que en todos los centros poblados se realiza el comercio de cueros por ejemplo Otorogo Panthera onca (US \$ 5.00). Entre las especies vendidas como mascotas predominan monos "choro" Lagothrix lagotricha y "maquisapa" Ateles paniscus y guacamayos Ara sp. El precio fluctúa desde US $\$ 7.00$ a 17.00 , dólares americanos.

En la Comunidad Nativa Santa Rosa y en el centro poblado Abujao se registraron la mayor cantidad de especies utilizadas, 20 y 21 especies respectivamente. En otros centros poblados el número de especies usadas disminuye rápidamente, que podría deberse a la cercanía al gran mercado que representa Pucallpa.
Valor de Uso de las especies.- Las especies de mamíferos más importantes con el mayor valor de diversidad de usos VDE con un valor de 0.835 fueron 6 , destacando el coto, sajino, 2 especies de venado, y entre las aves con un valor de 0.667 fueron 4 especies, destacando el guacamayo y el loro hablador. El venado colorado es la especie más importante entre los mamíferos con un valor de VDP de 0.308 y entre las aves destacan la garza blanca y el paujil con un valor de 0.1921 . En cuanto al uso más importante para el poblador de la cuenca VDU es el uso como alimento con un valor de 0.887 , seguido del uso como mascota con un valor de 0.500 (Tablas 3, 4 y 5).

\section{Discusión}

Los resultados encontrados son similares con trabajos realizados en otras partes de la Amazonía, como el caso de Escobedo y Ríos (2003), en el río Huallaga (Loreto, Perú), identificaron 19 familias de mamíferos representadas por: Tayassuidae, Tapiridae, Agoutidae y Atelidae. Entre las aves, las familias más representativas resultaron ser: Psittacidae y Cracidae. Por otro lado León (2006) afirma que la fauna silvestre neotropical generalmente ha sido utilizada con fines alimentarios, comerciales, ornamentales, ceremoniales y medicinales basándose en otros estudios (Bodmer \& Pezo 1999, Zapata 2001, Quijano-Hernández \& Calmé 2002, Milner-Gulland et al. 2003, Barrera-Bassols \& Toledo 2005). En la cuenca del río Abujao donde se realizó este estudio, los usos de la fauna silvestre son similares a los reportados en la literatura, siendo el principal producto la carne de monte.

$\mathrm{Al}$ respeto de uso como mascota en la cuenca del río Abujao, este uso se origina a través de encuentros fortuitos con animales silvestres o sus crías, también se realizan capturas utilizando trampas, o saqueando nidales y madrigueras para criar los cachorros, pichones o huevos y estos son colocados en los nidos de gallinas o patos que estén incubando, coincidiendo con lo descrito por Pautaos (2003).

Comparando resultados de usos medicinales con la literatura encontramos varias similaridades en uso de órganos reproductivos de monos, las cuales contienen fuertes dosis de hormonas que se emplean para fortalecer y activar la potencia viril. Escobedo y Ríos (2003) manifiestan que en el río Huallaga (Loreto, Perú) las comunidades indígenas utilizan la vértebra cervical de pecaríes, el cuerno de "venados" (Mazama gouazuobira) y "venado colorado" (Mazama americana) con fines medicinales para curar males espirituales "mal aire" según la cosmogonía del poblador local. Mientras que en el río Abujao para curar esta enfermedad se ha registrado solo el "venado colorado" (Mazama americana). Según trabajos realizados en comunidades neotropicales (Pèrez 2000, León 2006) que consideran que se están perdiendo ciertas prácticas ceremoniales y medicinales que estén vinculadas con la fauna silvestre. Al respecto León (2006) atribuye que una posible explicación de esto es la transfiguración ideológica de la población en algunos casos por la influencia de doctrinas religiosas.

En la cuenca del Abujao, la caza comercial también presenta diferentes usos comerciales de animales silvestres vivos o sus partes (carne, pieles y cueros), además de productos o despojos coincidiendo con (Cordero 1990, TCA 1995, Ojasti 2000, Escobedo \& Ríos 2003). Como en otras partes de bosques Neotropicos (Ojasti 2000) en la cuenca del río Abujao la caza comercial aporta bienes de cambio e involucra, además del cazador, un producto con un precio y a menudo una cadena de 
Tabla 5. Valores de diversidad para cada Uso (VDU) de las especies reportadas en la cuenca del río Abujao. Ucayali

\begin{tabular}{llcl}
\hline & Usos & Especies & VDU \\
\hline 1 & Alimentario & 55 & 0.887 \\
2 & Mascota & 32 & 0.500 \\
3 & Comercial & 29 & 0.419 \\
4 & Medicinal & 21 & 0.339 \\
5 & Ornato & 19 & 0.306 \\
6 & Mágico religioso & 4 & 0.065 \\
\hline
\end{tabular}

intermediarios entre el cazador y el comprador final y se practica en varias formas y escalas. Los resultados obtenidos sobre la comercialización de carne de monte son similares a los reportados por otros estudios en el ámbito del Neotrópico, donde se coincide que las especies más utilizadas para la comercialización de carne son: Agouti paca, Tayassu pecari, T. tajacu, Mazama sp., etc. En tanto el precio de venta de carne de mamíferos en la cuenca del río Abujao, tiene un precio promedio de S/. 6.00 nuevos soles, similar a lo reportado por Saldaña y Rojas (2003). Pero difiere con lo reportado por Escobedo y Ríos (2003), y Aquino et al. (2007), quienes registraron el precio promedio de la carne de monte de S/. 3.00. Ojasti (2000) manifiesta que la comercialización de la carne de monte es un artículo de primera necesidad y de alta demanda, ante todo en los caseríos, aldeas, campamentos mineros y otros centros poblados aislados por largas distancias o falta de vialidad. Los resultados obtenidos en este estudio sobre la comercialización de cuero coinciden con otros estudios realizados en el neotrópico donde las principales especies comercializadas son: Tayassu pecari, T. tajacu, Mazama sp. y Panthera onca (Saldańa \& Rojas 2003, Escobedo \& Ríos 2003, Ojasti 2000).

\section{Conclusiones}

La importancia social que tiene la fauna silvestre para los pobladores de la cuenca del río Abujao, está relacionada con las diferentes formas de aprovechamiento que de estas se realiza. Entre las categorías de uso el más importante fue el alimenticio. Según índices predeterminados, el valor de diversidad para uso alimentario fue 0.887 .

En segundo puesto esta el uso como mascota y en tercer lugar el uso comercial. Existen particularidades en el uso medicinal de las especies; la parte más utilizada resultó ser la grasa y la impotencia fue la enfermedad de mayor significación, mientras que para el uso artesanal y ornamental las plumas fueron las partes más utilizadas y la aplicación de mayor significancia fue de adornos de casa. Las especies con mayor número de usos, pertenecen al grupo de mamíferos, siendo 7 especies las que encabezan la lista con un Índice de VDE de 0.833. El valor de Diversidad de Uso de Partes y Productos de Cada Animal (VDPA), proporcionó información sobre el aprovechamiento de cada especie, encontrándose que el "venado colorado" Mazama americana fue la especie de la cual más partes o productos se aprovechan, 8 partes en total y teniendo un VDPA de 0.296.

Las tradiciones de usos rituales, religiosos y ornamentales se están perdiendo porque la generación joven no guarda tanto estos conocimientos. Este estudio representa un gran rescate del etnoconocimiento que forma una base muy importante para la conservación de los conocimientos para el mundo y para los mismos centros poblados.

\section{Literatura citada.}

Álvarez J. 2007. Comunidades locales, conservación de la avifauna y de la biodiversidad en la Amazonía peruana. Congreso internacional de Ornitología. Revista Peruana de Biología. 14(1): 145-150. DOI http://dx.doi.org/10.15381/rpb.v14i1.2181

Aquino R., T. Pacheco, M. Vásquez. 2007. Evaluación y valorización económica de la fauna silvestre en el río Algodón, Amazonía peruana. Iquitos, Perú.

Barrera-Bassols, N. \& V. Toledo. 2005. Ethnoecology of the Yucatec maya: Symbolism, knowledge and management of natural resources. Journal of Latin American Geography. 4(1):9-41. DOI http://dx.doi.org/10.1353/lag.2005.0021

Bisbal E.F.J. 1994. Consumo de fauna silvestre en la zona de Imataca, Estado Bolívar, Venezuela. Interciencia.

Bodmer R. 1993. Manejo de fauna Silvestre con las comunidades locales: el caso de la Reserva Comunal Tamshiyacu-Tahuayo. Manuscrito, caso de estudio 12b, Univ. de Florida.

Bodmer R.E., Fang, T.G. \& L. Moya. 1988. Ungulate management and conservation in the Peruvian Amazon. Biol. Conservation 45: 303-310.

Bodmer R. E. \& E. Pezo. 1999. Análisis Económico de la Venta de Carne de Monte y Exportación de Pieles en Loreto-Perú. En: T. Fang, O. Montenegro, R. Bodmer (eds). Manejo y Conservación de la Fauna Silvestre en América Latina. Memorias del 3er Congreso Internacional de Fauna Silvestre en la Amazonía realizado en Santa Cruz-Bolivia, 1997. La Paz. Edit. Visuall-Concepto Gráfico. 171-182 pp.

Centro de Investigación de la Frontera Amazónica - CIFA, 2009. Proyecto Abujao. Informe Anual 2008. Universidad Nacional de Ucayali, Facultad de Ciencias Agropecuarios, Pucallpa, Perú

Cordero R., G.A. 1990. Aprovechamiento de la fauna silvestre en Barlovento, Estado Miranda, Venezuela. Vida Silvestre Neotropical 2(2):70-74.

Escobedo A. \& C. Ríos. 2003. Uso de la fauna silvestre, peces y de otros productos forestales no maderables en las comunidades de las etnias Quechua y Achuar del Río Huasaga, Loreto-Perú. Tesis para optar el título profesional de biólogo. Facultad de Ciencias Biológicas, Universidad Nacional de La Amazonía Peruana. 199 pp.

Chirif A. 1983. Saqueo Amazónico. Ed. CETA. Iquitos.

González N.J. 1999. Análisis de las poblaciones de aves silvestres de importancia socio económicas en el sector meridional de la reserva nacional Pacaya-Samiria (Loreto, Perú) y bases para su manejo. Tesis Mg. Sc. Escuela de Post Grado, Universidad Nacional Agraria La Molina. Lima.

León P. 2006. Aprovechamiento de fauna silvestre en una comunidad aledaña a la Reserva de la Biosfera Los Petenes, Campe- 
che. Tesis de maestría. Maestría en Ciencias, especialidad de Ecología Humana. CINVESTAV-IPN. 108 pp.

Milner-Gulland E., E. Bennett \& S.A.M.W.M. Group. 2003. Wild meat: The bigger picture. TRENDS in Ecology and Evolution. 18(7):351-357. DOI https://doi.org/10.1016/S01695347(03)00123-X

Ojasti J. 1993. Utilización de la fauna silvestre en América Latina. Situación y perspectivas para un manejo sostenible. Guía FAO: Conservación 25:1-248.

Ojasti J. 2000. Manejo de Fauna Silvestre Neotropical. F.Dallmeier. (Ed.) SIMAB Series N 5 Smithsonian Institution / MAB Program, Washington, D.C, 290 p.

Phillips \& Gentry. 1993. The useful plants of Tambopata, Peru: I. Statistical hypothesis tested with a new quantitative technique. Economic Botany 47 (1): 15-32. DOI https://doi. org/10.1007/BF02862203

Pyhälä A. 2003. Productive Conservation in Amazonia: Institutions, Participation and Markets. PhD Thesis submitted to the School of Development Studies, University of East Anglia, U.K. http://ethos.bl.uk/OrderDetails.do?uin=uk. bl.ethos.405714

Quijano-Hernández, E. y S. Calmé. 2002. Patrones de cacería y conservación de la fauna silvestre en una comunidad maya de Quintana Roo, México. Etnobiología. 2:1-18.

Redford K.H. and J.G. Robinson. 1987. The game choice: Patterns of indian and colonist hunting in the Neotropics. American Anthropologist 89:650-667. DOI https://doi.org/10.1525/ aa.1987.89.3.02a00070

Saldaña J. Y T, Rojas. 2003. Consumo de Carne de Monte y su importancia en la alimentación del poblador de Jenaro Herrera, Loreto - Perú. MEMORIAS: VI Congreso Internacional sobre el Manejo de Fauna Silvestre en la Amazonía y Latinoamérica, Iquitos, Perú. http://www.comfauna.org/ wp-content/uploads/2012/PDFs-Manejofaunasilvestre/
Iquitos-2004/6_Conservacion-uso-y-manejo-de-faunasilvestre-por-comunidades/602-609_jsaldana_ConsumoCarneMonteImportanciaAlimentacion.pdf

Tratado de Cooperación Amazónica (TCA). 1995. Uso y conservación de la fauna silvestre en la Amazonia. Tratado de Cooperación Amazónica, Secretaria Pro-Tempore, Lima 35:1-216.

IIAP 2004. Informes finales del Proyecto "Conservación de la biodiversidad y manejo comunal de los recursos naturales en la cuenca del río Nanay, Loreto, Perú». Instituto de Investigaciones de la Amazonía Peruana. Informe técnico, Iquitos.

Zapata G. 2001. Sustentabilidad de la cacería de subsistencia: El caso de cuatro comunidades Quichuas en la Amazonia Nororiental Ecuatoriana. Mastozoología Neotropical. 8(1):59-66. 